\title{
EL PAPEL DE LAS LEYES GENERALES EN LA EXPLICACIÓN CAUSAL
}

Hempel ha insistido en que toda explicación debe incluir leyes generales, y en general los filósofos de la ciencia han aceptado esta tesis. Sin embargo, existe una amplia zona del conocimiento humano, una amplia zona de la conducta racional humana, en la que ofrecemos y aceptamos explicaciones sin apelar explícitamente a ninguna ley general. Piénsese, por ejemplo, en las explicaciones del sentido común, de la psicologia, la politica, la historia, la medicina. Tenemos, pues, el siguiente dilema: o Hempel y los filósofos de la ciencia están equivocados, o todas estas aparentes zonas del conocimiento son zonas oscuras en las que reina la pseudoexplicación y la charlatanerfa. Esta segunda posibilidad, es decir, el camino positivista, es falsa e indeseable. Falsa, porque presupone una idea a priori de la racionalidad, independiente de la racionalidad humana; indeseable porque, al proponer condiciones inalcanzables en importantes zonas de nuestras creencias, deja la mayor parte de nuestra acción al arbitrio de la irracionalidad. El exceso en los ideales de rigor conduce a un antiintelectualismo en la práctica. Pero tampoco podemos concluir simplemente que Hempel y sus seguidores se equivocan. Los argumentos en favor de la inclusión de leyes generales en la explicación no pueden dejarse de lado sin examen.

Podemos enmarcar este dilema en el siguiente planteamiento que se debe a A. Danto. Tenemos tres tesis que parecen verdaderas, pero son incompatibles entre si:

(1) Los historiadores logran a veces explicar por qué ocurrió un suceso.

(2) Toda explicación debe incluir leyes generales estrictas. (Es decir, deterministas, sin excepciones.)

(3) Las explicaciones que ofrecen los historiadores no incluyen leyes generales.

Como puede verse, el plańteamiento de Danto se refiere a la historia. Pero donde dice "historiadores" podemos escribir "médicos", "psicólogos", "politólogos", etcétera, sin que las oraciones resultantes sean menos verosímiles ni menos incompatibles. No discutiremos la tesis (3), pues su función en este planteamiento sería indicar las zonas del conocimiento en las que las explicaciones normalmente no incluyen leyes generales. Si esto no fuera cierto en todas las explicaciones históricas, habrfa de todas formas explicaciones convincentes de este tipo dentro del campo de la historia, y seguramente las 
hay dentro de las otras zonas del conocimiento humano que hemos mencionado. La precisión de la tesis (3) es la precisión del campo de vigencia del problema. El problema es, pues, que (1) y (2) son incompatibles (dada la verdad de (3)) y al mismo tiempo nos parecen obvias. Un tipo de solución consistirá en rechazar una de las dos, y explicar por qué nuestras intuiciones estaban equivocadas. Pero podemos también reinterpretar las tesis de manera que salvemos su obviedad y desaparezca su incompatibilidad. Así, Danto clasifica las soluciones que se han dado al problema de acuerdo con estos cuatro renglones:
A.
(1) es falsa,
(2) es verdadera.
$\boldsymbol{B}$.
(1) requiere reinter- pretación,
c.
(I) es verdad,
(2) es verdadera.
(2) requiere reinterpre- tación.
D.
(1) es verdad,
(2) es falsa.

\section{I}

El caso extremo de la solución $A$ sería el de los positivistas iconoclastas a los que me referi al comenzar este trabajo. Los historiadores no explican; la explicación es la única forma de entender por qué ocurrió algo. Danto clasifica aqui a los idealistas históricos (Croce, Dilthey, Collingwood) cuya posición es menos extrema debido a que estos filósofos restringen el sentido de "explicacion", pero aceptan otras formas, que no son explicaciones, de entender por qué ocurrió algún acontecimiento. El conocimiento del mundo está dividido en dos grandes sectores incomunicables: las ciencias naturales (Naturwissenschaften) y las ciencias del espiritu (Geisteswissenschaften). Las ciencias naturales son las únicas que explican, y lo hacen mediante leyes deterministas estrictas. Las ciencias del espíritu; en cambio, no podrán explicar los acontecimientos de los que tratan porque estos acontecimientos son actos libres del hombre, y la libertad no puede estar sujeta a leyes deterministas. Los actos humanos, únicos e irrepetibles, no pueden explicarse por leyes generales y deben, en cambio, interpretarse a la luz de propósitos, motivos, deseos, creencias, todos ellos estados mentales inobservables. El historiador puede entender - no explicar - mediante la aprehensión empática del funcionamiento interno de la mente. 
Pareceria que aquí nos encontramos con un simple problema verbal: no queremos aplicar la palabra "explicación" a este tipo de explicaciones. El asunto, sin embargo, es más importante: la idea de estos filósofos es que la explicación naturalista y la explicación mentalista (interpretación) no tienen ninguna relación entre sí $\mathrm{y}$, además, que los actos humanos no son explicables mediante leyes generales.

La aprehensión empática es un procedimiento muy conocido: ponernos en la situación, en los zapatos del sujeto cuyos actos queremos entender. Si imagino que he perdido una batalla decisiva, y que soy el único responsable, entiendo que se me ocurra la posibilidad de suicidarme, entiendo por qué podría tomar esa decisión, y así me explico el suicidio del general González Salas. En cambio, si me dijeran que el general se suicidó porque se sacó un cuadro de Picasso en una rifa, la explicación me parecerá absurda porque no me imagino cómo semejante situación pudiera inducirme al suicidio (aun si el general hubiera detestado la pintura de Picasso). La razón por la que no se mencionan leyes en la historia es porque no se pretende explicar los hechos históricos (en el sentido de las ciencias naturales) sino entenderlos. Pedir explicaciones a los historiadores es no entender la naturaleza de su disciplina y cometer el error melafísico de pensar que los hombres están determinados como los demás seres, cuando en realidad son libres.

Tenemos aquí un dualismo de los acontecimientos. Hay acontecimientos naturales $y$, por tanto, determinados, que pueden explicarse, y acontecimientos libres, humanos, que sólo pueden interpretarse, pero no explicarse. La posición de los idealistas históricos se basa en la creencia de que la libertad y el determinismo son incompatibles: un acto cuya ejecución sea necesaria por seguirse de una ley estricta, no quedará al arbitrio del agente, no habrá la posibilidad de que no quiera ejecutarlo. Esta idea, por otra parte, puede conducir a la conclusión paradójica de que los actos libres no tienen causas. Si la relación causal no puede darse sin una ley, se dirá, los actos humanos no tienen causas, sino que se entienden por las razones del agente, sus deseos, creencias, propósitos, etcétera. La conclusión es paradójica porque si los actos del agente no tienen causas, es decir, si ningún suceso anterior los ha producido, apelar a los estados mentales del agente resulta inútil: esos estados no explicarán ninguna conexión causal.

\section{II}

Hempel propone una solución del tipo $B$ : es necesario reinterpretar la tesis de que los historiadores ofrecen explicaciones. En su artículo El papel de las leyes generales en la historia ataca la tesis del idealismo histórico. Hempel cree 
en una sola realidad y en una sola ciencia. Las leyes generales cumplen funciones análogas en la historia y en las ciencias naturales. La explicación consta de dos partes, un "explanandum", es decir, la oración que describe el acontecimiento que queremos explicar, y un "explanans" constituido por las oraciones explicativas. Éstas son de dos tipos, un conjunto de leyes generales y un conjunto de condiciones particulares del caso. Para que una explicación sea aceptable, debemos tener buenas razones para creer en todas las oraciones que la forman. Si somos empiristas, como Hempel, diremos que las leyes generales deben estar debidamente confirmadas por la experiencia. Por otra parte, el explanans tendría que implicar lógicamente la verdad del explanandum. Si se cumple esta condición, resultará imposible que se dé la verdad del primero sin la del segundo. Este requisito concuerda con una inclinación intuitiva: para que nos satisfaga la explicación tenemos que dar razones que excluyan la posibilidad de que hubiera ocurrido otra cosa en lugar de la que queremos explicar. Entender sería ver "por qué esto tenía que ser asi". Pedir la relación de implicación lógica es pedir que todas esas razones sean explícitas.

Hempel distingue la explicación genuina, la que cumple con los requisitos formales, de la pseudoexplicación, que es explicación sólo en apariencia. En la pseudoexplicación encontramos que falta alguna de las condiciones a las que nos hemos referido: no se da la relación deductiva (implicación lógica) entre explanans y explanandum o las leyes aducidas no pueden ponerse a prueba en la experiencia. Esta distinción separa las pseudoexplicaciones de las explicaciones genuinas que son inadecuadas porque alguna de las oraciones del explanans resulta falsa. Con respecto a las pseudoexplicaciones no tiene caso, o no tiene sentido, preguntarse si las oraciones que forman el explanans son verdaderas, puesto que no se han cumplido condiciones esenciales previas.

Con todo, casi nunca encontramos explicaciones completas según las instrucciones de Hempel. Esto se debe a que decimos sólo lo que hace falta: lo sabido lo damos por supuesto. La mayoría de las explicaciones son, pues, elipticas. Pero hacerlas explícitas no es inútil. Al expresarlas en su totalidad podemos examinarlas, podemos averiguar si son falsas o si son pseudoexplicaciones. Esta idea nos permite reinterpretar (1) y reconciliar este enunciado con (2) y (3). Los historiadores ofrecen explicaciones elípticas y por esta razón no mencionan leyes generales, pero apelan a ellas tácitamente.

Tenemos también casos en los que no se pretende haber llegado a la explicación en sentido estricto, sino solamente a un esbozo de explicación. La vaguedad con que se indican las leyes generales y el tipo de condiciones particulares puede deberse a un intento de'aparentar tener lo que no se tiene, pero puede ser también un primer bosquejo de lo que quiere encontrarse. 
Propuestas así, como esbozos, estas leyes y condiciones se irían afinando y completando mediante la investigación empirica. Las leyes resultarán más precisas y su grado de confrontabilidad empírica tendrá que ser mayor. Esta idea puede contribuir también a la reinterpretación de (1). Muchas de las explicaciones de los historiadores podrian ser solamente bosquejos de explicación.

Los idealistas se equivocan al suponer que los hechos únicos e irrepetibles no pueden explicarse por leyes generales. El hecho de que un suceso sea irrepetible y único sólo indica que no habrá otro que tenga todas sus propiedades, pero esto no impide que tenga algunas propiedades en común con otros sucesos. Pues bien, ninguna ley considera en su antecedente todas las caracteristicas de un suceso, sino sólo algunas que pueden compartir varios sucesos únicos e irrepetibles. Los sucesos físicos son tan únicos y tan irrepetibles como los actos humanos; las leyes que los explican no se refieren a todas sus caracteristicas.

Pero ¿es cierto que todas las explicaciones apelan a leyes sin excepciones? Algunos autores nos dicen que en la vida ordinaria explicamos los acontecimientos apelando tácitamente a generalizaciones burdas en las que la expresión "en general" quiere decir "casi siempre" y no "siempre". Nada obsta para que los historiadores ofrezcan explicaciones causales semejantes (M. Scriven). Otros autores, como Ernest Nagel y después el propio Hempel, han sugerido la posibilidad de explicaciones probabilísticas basadas en leyes probabilísticas: "es altamente probable que cuando ocurre un suceso del tipo $c$, ocurra un suceso del tipo $e$ ". De esta forma se propone una solución $C$ en la clasificación de Danto. El enunciado (2) debe ser reinterpretado, ya sea diciendo "algunas explicaciones incluyen leyes no estrictas" o "algunas explicaciones sólo incluyen leyes probabilísticas". Más adelante criticaré esta idea.

La comprensión empática de los idealistas históricos no explica absolutamente nada, no nos permite entender por qué ocurrió el suceso que queremos explicar. Claro, el historiador podrá imaginarse a sí mismo en la situación del agente histórico cuyas acciones quiere entender, y podrá seguramente imaginarse lo que haria en esas circunstancias. Esto ayudará a que se le ocurran hipótesis psicológicas explicativas. Pero estas hipótesis solo serán explicativas si tienen fundamento empírico y si el explanandum puede deducirse de ellas, o, si se pretende dar una explicación probabilística, será necesario que se ofrezca una ley probabilística adecuada. Pero las explicaciones habrán de juzgarse por estas caracteristicas objetivas y no por su capacidad de hacernos sentir que nosotros hubiéramos podido hacer lo mismo que el agente cuyas acciones queremos explicar.

Tenemos, pues, varias sugerencias para resolver el problema de las explicaciones que no apelan a leyes (cuando no se trata de pseudoexplicaciones): 
(i) son elfpticas, (ii) son esbozos de explicación, (iii) son explicaciones probabilfsticas. Ninguna de las tres me parece satisfactoria. El general González Salas se suicidó porque Pascual Orozco lo derrotó en Rellano. Esta explicación nos parece correcta, pero ¿estamos apelando tácitamente a una ley estricta y general? No se ve claramente cuál sería. La explicación es satisfactoria aunque los generales rara vez se suiciden cuando pierden una batalla, por más responsables que se sientan. No estamos, pues, apelando tácitamente a una ley. Pero tampoco se trata de un esbozo de explicación, porque esta explicación no se propone como la primera formulación de una ley estricta que intentaremos descubrir en el futuro. Más adelante, al referirme a la tesis de la anomalia de lo mental, encontraré razones para pensar que ninguna reformulación del esbozo de ley "Todos los generales que pierden una batalla se suicidan" se convertirá en una ley estricta. Esto no quiere decir que no pueda haber ninguna ley estricta que relacione la pérdida de la batalla (ese suceso) con el suicidio del general; el problema es que esa ley, de existir, no se parecerfa en nada al esbozo apuntado. (Esto quedará más claro cuando explique el análisis de Davidson de los enunciados causales.) Pero tampoco funciona la sugerencia probabilista: ¿se suicidó el general porque la probabilidad de que un general que pierde una batalla se mate es de .001? Aun en los casos con alta probabilidad, la cosa no parece clara. El capitán del Titanic se hundió con su barco porque se sintió obligado a hacerlo, según la tradición de los capitanes de barco, no porque la probabilidad de que un capitán se hunda con su barco sea de .99 .

Más adelante mostraré que si aceptamos la forma lógica de los enunciados causales singulares que nos propone Davidson, tenemos otra forma de aceptar la solución $C$. Pero antes voy a ocuparme del último renglón de Danto.

\section{III}

En la posición $D$ se encontrarían los críticos radicales de Hempel, es decir, quienes niegan que toda explicación deba incluir leyes generales. Danto propone el ejemplo de William Dray. Yo me referiré, en cambio, a Elisabeth Anscombe, cuya posición es más clara, o más radical.

Tál vez el lector comparta con Aristóteles, Espinosa, Hobbes, Hume, Kant, Mill y Russell la intuición de que, si $c$ es la causa de $e$, una vez que $c$ aconteció no hubiera sido posible que $e$ dejara de suceder. En otras palabras, la causa tiene un vínculo de necesidad con el efecto: dada la causa, el efecto necesariamente ha de ocurrir. Para justificar esta intuición suele acudirse a la pregunta retórica: ¿en qué sentido podria entenderse que $c$ causó $e$ si se admite la posibilidad de que hubiera ocurrido $c$ sin que llegara a pasar $e$ ? 
Pero con ello no hacemos más que apelar a la convicción misma que queríamos justificar.

Obviamente esta intuición permite diversas interpretaciones y teorías, dado que la noción de necesidad puede entenderse en formas distintas. Pero en todas ellas encontraremos una idea común:

Si un efecto sucede en cierta ocasión, y un efecto similar no acontece en alguna ocasión que parece similar, debe haber alguna diferencia entre las dos ocasiones que nos ha pasado inadvertida.

Esta doctrina está implícita en la idea de que dada $c$ tenía que ocurrir e. Porque, si en algunos casos el efecto se produce y en otros no, ¿qué podria querer decir "tenfa que ocurrir"? La única solución está en suponer que los casos son diferentes.

Hemos dicho que este vinculo de necesidad admite diferentes interpretaciones. Hume, por ejemplo, criticó la doctrina de Hobbes y los racionalistas en el sentido de que se trataba de una relación lógica o conceptual. Hobbes afirma que cuando se da la causa "no puede entenderse sino que se. produce el efecto". La idea es que si conocemos una causa no podremos negar que se producirá el efecto sin contradecirnos lógicamente. Hume examina los hechos que nosotros relacionamos causalmente y no encuentra ninguna contradicción en la idea de que los primeros hubieran podido ocurrir sin los segundos. La calda de una piedra, por ejemplo, no puede deducirse del análisis de los conceptos involucrados en la descripción del suceso y de su causa. Si hay una relación de necesidad entre causa y efecto, ésta no será una relación lógica.

(Anscombe tiene razón al decir que esto sólo pudo ser novedad en la República de las Letras y no para la humanidad en general. Los esfuerzos de Hume por mostrar la inexistencia de una contradicción que el común de la gente, "siempre más que dispuesta a creer en milagros, maravillas y lusus naturae", jamás habfa imaginado, soblo se explican en atención a las teorfas filosbficas de sus predecesores.)

La idea de que no habla necesidad lógica entre causas y efectos no liberó a Hume ni a la tradición posterior de la convicción de que existlà algún vinculo de necesidad. Asi, Hume, lejos de abandonar el prejuicio de la nécesidad, ideó una nueva forma de interpretarlo y justificarlo que sigue vigente hasta la fecha entre filósofos de la ciencia. La mente humana, que efectivamente no ve una relación necesaria entre los sucesos y sus causas, observa, sin embargo, la constante conjunción entre ciertos tipos de sucesos y ciertos otros. Siempre que se calienta el agua hasta cierta temperatura, hierve. De esta manera la mente construye hipótesis acerca de regularidades de la naturaleza, que expresará en generalizaciones que no admiten excepciones. Estas gene- 
ralizaciones, por tanto, pretenden descubrir relaciones de necesidad en la naturaleza: si se dan las condiciones establecidas ("condiciones iniciales"), ya que no hay excepciones, tendrá que ocurrir el efecto previsto. Estas generalizaciones, entonces, son leyes deterministas: dadas las condiciones iniciales, queda determinado de antemano un resultado especifico, ya no por necesidad lógica, sino, ahora, por necesidad física. tsta implica un análisis complejo de los enunciados causales singulares. Cuando afirmamos que cierto suceso $c$ causó $e$, decimos varias cosas: que los dos sucesos ocurrieron, que "si $c$ ocurre, entonces $e$ ha de ocurrir" es un caso particular de una generalización sin excepciones y que por ello, habiendo ocurrido $c$, tenfa que ocurrir $e$.

Según Anscombe, es fácil mostrar que no debe asociarse la causalidad con ningún género de necesidad. Su primer argumento se basa en la consideración de la forma en la que conocemos la verdad de los enunciados causales. "Es mucho más fácil encontrar con certeza las causas, una vez que conocemos los efectos, que predecir los efectos, dadas las causas. Con múcha frecuencia sabemos cuál es la causa sin saber si existe una generalización sin excepciones adecuada al caso." Por ejemplo, sabemos que la causa de muchas enfermedades contagiosas ha sido el contacto con un enfermo, pero en la mayor parte de los casos, ningún médico podrá predecir el contagio a partir del contacto con el enfermo. Sabemos, pues, de causas y efectos sin conocer generalizaciones que nos justifiquen en la forma que Hume sugiere.

E. Anscombe atribuye a la noción de causa un significado que nada tiene que ver con la necesidad. El efecto proviene, es consecuencia, se deriva de su causa, surge de ella, se origina en ella, la causa lo produce. El análisis en términos de necesidad no sólo añade algo que no se encuentra en el concepto, sino que pasa por alto la idea esencial: no nos dice que el efecto provenga de la causa porque la relación de necesidad no implica la de proveniencia. Si una mujer dio a luz a su segundo hijo, es necesario que haya parido antes a otro, pero el primer parto no proviene del segundo.

El verbo "causar" no se usa con mucha frecuencia. En general, preferiremos expresar la causalidad mediante verbos causales particulares como matar, rascar, infectar, dar, empujar, quitar, mojar, levantar, comer, quemar, mover, aplastar, arruinar, hacer ( $v$. gr. ruidos, pajaritas de papel), etc. Hume habła pensado que jamás veremos la eficiencia, la proveniencia, la causalidad, porque no podemos ver la necesidad y porque aceptaba planteamientos cartesianos que aqu no discutiré. Pero si no atendemos a esos prejuicios cartesianos y no entendemos la causalidad como necesidad, no tendremos por qué poner en duda que hemos observado acciones como matar, mojar o empujar.

Hasta aqui, las tesis de E. Anscombe. ¿Cómo se relacionan con la solución $D$ del esquema de Danto? De su tesis principal, que resumo a continuación, no se sigue que haya explicaciones que no incluyan leyes estrictas (no se sigue la falsedad de (2)): 
Tesis I: Las oraciones causales singulares, como " $c$ causó $e$ ", no implican una relación de necesidad. No es cierto que si $c$ causa $e$, al suceder $c$ se cancele la posibilidad de que $e$ no ocurra. En particular, no es cierto que de " $c$ causa $e$ " se siga la existencia de una ley universal que conecte $c$ con $e$.

Sin embargo, quien sostenga esta tesis defenderá también, muy probablemente, una doctrina de la que se sigue la falsedad de (2) y la posibilidad de la verdad de (1):

Tesis II: Cuando decimos que un suceso $c$ causó un suceso $e$, en muchos casos habremos dado una explicación de por qué ocurrió e, aunque no hayamos mencionado ninguna ley general. (La explicación rezaría: "aconteció $e$ porque sucedió $c^{\prime \prime}$.)

Digo que quien sostenga la primera defenderá probablemente la segunda porque de lo contrario su tesis carecería de interés. El conocimiento de las relaciones causales entre los acontecimientos del mundo es importante para nosotros porque nos permite entender cómo se producen, cómo llegan a ocurrir los sucesos. La estructura causal de la realidad es interesante debido a que es explicativa. Acabo de usar la expresión "cómo se producen los acontecimientos"; igualmente pudiera haber dicho "surgen", "se originan", etcétera; es decir, habría podido usar cualquiera de los términos que mejor interpretan el sentido de la causalidad según E. Anscombe. No es necesario insistir en la frecuencia con que aparecen verbos causales particulares en las explicaciones cotidianas.

\section{IV}

Del análisis de Davidson de los enunciados causales singulares, y de la Tesis II, podría seguirse una solución del tipo $C$, es decir, una solución que reinterprete la tesis de que toda explicación debe incluir leyes generales estrictas.

No es lo mismo decir cuál es la forma lógica de un enunciado que analizar los conceptos involucrados. Así, por ejemplo, describir la forma lógica de "Félix Díaz era sobrino de don Porfirio" podría consistir simplemente en la afirmación de que "era sobrino de" es una relación que se predica de las entidades referidas por las expresiones referenciales "Félix Díaz" y "don Porfirio", en ese orden, sin explicar el contenido de la relación. (Russell sugirió que la estructura lógica de esta oración es más compleja, pero discutir aquí la teoría de los nombres propios nos llevaría demasiado lejos.) Al decir esto, no analizamos el contenido de la relación; tampoco decimos qué significan 
o cómo refieren los nombres propios. Sin embargo, no podrenos justificar un análisis semejante sin decir a qué tipo de entidades pueden referirse las expresiones referenciales, es decir, entre qué clase de entidades se predica la jelación.

Hemos visto que en las predicaciones causales ordinarias se prefieren verbos causales especificos. Sin embargo, tomaremos como enunciados causales singulares a los enunciados más sencillos en los que aparece la palabra causa. Así, serian ejemplos de enunciados causales singulares: "El resbalón que dio el general Hernández el 25-XII-68 causó su muerte", "La explosión que ocurrió en la presa causó la inundación", "La inundación causó la ruina de las cosechas". Davidson propone un análisis muy simple de estos enunciados: tienen la misma forma lógica que "Félix Díaz era sobrino de don Porfirio", que " $3<5$ " y que "Hugo Hiriart es primo del Goyo Cárdenas"; todos estos enunciados predican una relación entre un par de entidades. Pero ¿qué clase de entidades pueden tener la relación causal? En otras palabras, ¿qué clase de objeto puede ser la referencia de "el resbalón que dio el general Hernández" o "la muerte del general Hernández"? La respuesta de Davidson no es nueva: afirma, con muchos filósofos del pasado, que la relación causal se da entre sucesos, acontecimientos o, si debe tolerarse la palabra, eventos. Lo novedoso en el caso de Davidson consiste en dos cosas. En primer lugar, la justificación de su tesis se da en el marco de una teorfa de la forma lógica más clara y explícita. En segundo lugar, Davidson se preocupa por las consecuencias filosóficas de este análisis; muestra que ciertos errores filosóficos importantes se deben al descuido en cuanto a la forma lógica de estos enunciados.

John Stuart Mill, por ejemplo, objeta la versión de la muerte del general Hernández que pudo aparecer en su obituario: "El general murió a consecuencia del resbalón que dio al pisar una cáscara de plátano el 25-XII-68." Según Mill, el resbalón no pudo ser toda la causa, porque no todo el que se resbala al pisar una cáscara de plátano muere. Faltan muchos elementos: "la circunstancia de su peso", que se resbaló en una escalera de mármol, que sus huesos no eran de aluminio, etc. La idea de Mill es que la causa está constituida por la totalidad de las circunstancias que hicieron necesario el efecto, o sea, por el cumplimiento de un conjunto de condiciones que nos permitan establecer una generalización sin excepciones según la cual tenía que ocurrir el efecto. Esta interpretación produce inmediatamente un problema al que ya nos hemos referido: ningún enunciado causal de los que normalmente consideramos verdadero sería completo en el sentido de Mill, y no sabemos, en la mayor parte de las cosas, cómo tendríamos que completarlo. Ahora bien, es imposible suponer que el análisis de Mill es correcto y que los hablantes normales, los usuarios del concepto, lo entienden de otra manera. Pero, si lo entendieran a la manera de Mill, estarían equivocados casi todas las veces que 
lo usaran, y tendrian creencias raras como la de que todo el que se resbale con una cáscara de plátano morirá, lo cual es falso.

¿Cómo pudo, sin embargo, llegar Mill a semejante conclusión? La idea de la condición necesaria y la elaboración de Hume, llevan a Mill a la opinión de que la causa tiene que ser condición suficiente del efecto. Más allá de que esto sea verdadero o falso, Mill lo hace jugar en un espacio conceptual nebuloso. El famoso resbalón no fue condición suficiente de la muerte del general, nos ha dicho Mill, porque si el general hubiera pesado lo mismo que una mosca, no hubiera muerto. El lector habrá comenzado ya a sospechar dónde se encuentra la confusión. Si las causas son sucesos, ¿puede un suceso ser una condición? Parece más claro decir que los sucesos cumplen condiciones y que, si son causas, cumplirán con una conjunción de condiciones que son suficientes para que ocurra otro suceso de cierto tipo que será su efecto. Si el resbalón fue la causa (y la doctrina de Hume es correcta), ese resbalón debe haber cumplido con ciertas condiciones que ejemplifican una generalización sin excepciones; el resbalón es, pues, la causa completa aunque no hayamos mencionado todas esas condiciones al referirnos a él.

Para que lo anterior resulte claro, tal vez convenga recordar la distinción que se asocia al nombre de Frege entre el sentido de una expresión y su referencia. Dos expresiones pueden tener la misma referencia y sentidos diferentes. Ast, "el lucero matutino" y "el lucero vespertino" se refieren al mismo planeta y tienen sentidos diferentes. Estas expresiones, lo mismo que "el resbalón que se dio el general Hernández cuando gritaba 'Viva la libertad" ", se refieren a la entidad que cumpla con las condiciones que establece su sentido. Si, por ejemplo, el general jamás hubiera gritado "Viva la libertad" o si se hubiera resbalado gritando "Muera Franco", la expresion anterior no se referiria a ningún suceso. Ahora bien, las condiciones mediante las cuales una expresión se refiere a un suceso no tienen por qué coincidir con las condiciones que nos permiten (si esto es posible) subsumir este suceso bajo una generalización estilo Hume. Al decir que el resbalón fue la causa, nos referimos al mismo suceso al que nos referimos con la larga expresión que Mill hubiera preferido (la que enumerarla todas las condiciones del suceso que garantizarian el efecto) y que equivocadamente llamaba "la causa completa".

El análisis de Davidson nos obliga a plantear la doctrina de Hume en una nueva forma y quita fuerza al primer argumento de $\mathbf{E}$. Anscombe. Si Hume tiene razón, los enunciados causales singulares implicarfan la existencia de una generalización que garantizarfa el nexo de necesidad, pero no implicarfan ninguna generalización en particular. Según esto, podrłamos tener razones para creer en la verdad de enunciados causales ( $y$ aun de explicaciones causales) sin disponer de alguna generalización aplicable al caso. Estas razones, empero, tendrán que convencernos de que existe una generalización de ese tipo, aunque no sepamos cómo podría formularse. Asf, si podemos 
saber, como alega Anscombe, que el contacto con un enfermo fue la causa del contagio, sin poder formular una generalización sin excepciones, no podremos concluir de aquí que nuestro enunciado causal no implique la existencia de alguna generalización. Es más, la tesis de $\mathrm{E}$. Anscombe de que podemos observar la eficiencia sugiere la posibilidad de que esas observaciones sean buenas razones para creer en la existencia de una generalización que no conocemos, dadas, claro, ciertas creencias de orden general.

La argumentación de quien siguiera a Davidson en el contexto que nos ocupa podría resumirse asf: (i) Es importante separar la cuestión de la forma lógica del análisis del contenido de un concepto. Es verdad que lo segundo no puede hacerse con entera claridad sin lo primero, pero podemos estar de acuerdo en la forma lógica de un enunciado sin coincidir en el análisis del contenido de alguno de sus conceptos. Hempel no atiende con cuidado a la forma lógica de los enunciados y produce un análisis confuso. (ii) Forma lógica. Los enunciados causales singulares, es decir los enunciados de la forma gramatical " $c$ causó $e$ " tienen la forma lógica $C c e$, es decir, afirman una relación entre dos objetos. Los objetos relacionados son sucesos o acontecimientos; no son cuerpos físicos, ni hechos o situaciones objetivas. Esto quiere decir que la causa no puede ser, como quieren Mill y Hempel, un conjunto de condiciones. Un suceso puede cumplir con ciertas condiciones, pero no es un conjunto de condiciones. (iii) Análisis del concepto. Los enunciados causales singulares implican la existencia de una ley que conecte dos sucesos llamados causa y efecto. Parte de su contenido, entonces, es que esa ley existe. Pero no implican, no es parte de su contenido, cierta ley en concreto. (iv) Las dos tesis anteriores, en el contexto de la distinción fregeana entre sentido y referencia, nos permiten concluir que para que un enunciado causal sea verdadero no es necesario que los términos mediante los cuales nos referimos a los sucesos involucrados, nos permitan derivar lógicamente el enunciado causal de una ley estricta, sino solamente que los sucesos en cuestión tengan caracteristicas conocidas o desconocidas, recogidas o no en los términos singulares que usamos para referimos a ellas, que los conviertan en un caso de aplicación de alguna ley estricta, conocida o desconocida. (v) Puesto que los enunciados causales singulares implican la existencia de una ley, sólo se justifica que los creamos o afirmemos si tenemos razones para creer que la ley existe. Ahora bien, ¿̇en qué pueden consistir esas razones? Lo importante es rechazar la idea de que sólo si podemos formular esas leyes podremos concluir que existen. Podemos, pues, tener buenas razones para creer en la verdad de enunciados causales singulares y podemos legitimamente creer en ellos, sin que estemos obligados a ofrecer una ley general como justificación. Ni siquiera estamos obligados a tener razones para concluir que si hacemos tales y cuales investigaciones empiricas podremos encontrar las leyes en cuestión. Es posible que no tengamos la menor idea de cómo pudieran ser esas leyes. El haberse 
enterado de que perdió la batalla causó el suicidio del general, pero no tenemos la menor idea de cómo podrán ser las leyes que conecten estos sucesos. Tal vez sólo puedan formularse en una neurofisiología del futuro que se parecerá poco a la actual. Si añadimos a estas ideas la Tesis II, podríamos concluir que no toda explicación causal que no ofrece leyes es una pseudoexplicación, ni un esbozo de explicación. Pero tenemos que explicar la Tesis II un poco más, para poder decir no sólo que los enunciados causales pueden ser verdaderos y que podemos afirmarlos sin que conozcamos ninguna ley, sino además, que son explicativos. Como ya había sugerido, estas ideas nos permitirán justificar una posición $C$ en la clasificación de Danto: (1) es verdad, pero (2) requiere reinterpretación. Los historiadores explican, pero no toda explicación causal debe ofrecer leyes generales estrictas. (2) se reinterpreta, más que negarse simplemente, porque la explicación implica la existencia de una ley general estricta.

Cuando Hempel habla de la causalidad sigue la tradición de Hume y Mill. Recoger sus ideas es útil en este momento, porque nos permite ver con claridad cómo la confusión entre causas y condiciones es un obstáculo para la aceptación de la Tesis II. Sigo sus razones:

(i) "Una causa es un conjunto más o menos complejo de circunstancias y sucesos que podría describirse por un conjunto de enunciados $C_{1}, C_{2}$, $\ldots C_{k}$ "

(ii) El principio "misma causa, mismo efecto" indica que implícitamente la explicación causal afirma la existencia de leyes generales. Afirmar que $C_{1}, C_{2} \ldots C_{k}$ causaron conjuntamente $E$ es afirmar que siempre que ocurran sucesos y condiciones del mismo tipo que $C_{1}, C_{2}, \ldots C_{k}$ ocurrirá un suceso del mismo tipo que $E$, y esto expresa una ley general.

(iii) Esto quiere decir que la relación entre los factores causales y el efecto queda reflejada en el esquema nomológico deductivo de explicación: la explicación causal es, por lo menos implícitamente, nomológico-deductiva.

(iv) En la mayoría de las explicaciones causales las circunstancias mencionadas no son completas. Supongamos que decimos que $a$ causó $b$ (y éstos son sucesos individuales). Aquí se afirma tácitamente una ley de esta forma: dadas ciertas condiciones, a todo suceso de tipo $A$ (del cual $a$ es un caso particular) sucede un evento de tipo $B$ (del cual $b$ es un caso particular). En el enunciado " $a$ causó $b$ " no siempre se mencionan las circunstancias relevantes.

Supongamos que $a$ es la calda de un cigarro encendido en un montón de paja y $b$ es el incendio de ese montón de paja. Decir que $a$ causó $b$ implica que se dieron un conjunto de circunstancias tales que siempre que se dan y hay un suceso de tipo $A$ ocurre un suceso del tipo $B$.

(v) De aqui Hempel concluye que en la medida en que el enunciado causal individual deje condiciones sin mencionar y por tanto leyes indispensables para la explicación, en la medida de la vaguedad con que las evoque, 
en esa medida se aproximará en valor informativo (explicativo) a un pergamino que afirma la existencia de un tesoro en alguna parte no especificada. Su utilidad e importancia aumentará conforme se especifique el lugar con mayor claridad. Cuando las leyes y condiciones permanecen en gran medida indefinidas, un enunciado causal es más un programa o un esbozo para una explicación; puede verse también como una hipótesis de trabajo.

Esta comparación es incorrecta. En el caso de la explicación nomológicodeductiva, efectivamente, la ausencia de condiciones y leyes restará valor informativo a la explicación hasta llegar a asemejarse al pergamino inútil. Pero no todas las explicaciones son nomológico-deductivas. Si el análisis de Davidson es correcto, los enunciados causales no incluirán necesariamente condiciones necesarias o suficientes. Entendemos perfectamente verbos causales como "contagiar" o "matar" sin conocer las condiciones estrictas en las que esto sucede. Los enunciados causales son explicativos en la medida en que nos dicen que cierto suceso $c$ causó cierto suceso $e$. La situación no es semejante a la del pergamino porque aquí sí se nos dice dónde está el tesoro: se nos informa cuál es el suceso responsable del efecto. Más adelante veremos que no todas las descripciones del mismo suceso son igualmente explicativas. Pero tampoco son igualmente informativas todas las descripciones de un individuo, y a veces esto depende de lo que sepamos. ("E1 autor del Quijote es la persona que pisó este escalón exactamente en t.") $S i$ durante años nos preguntamos por qué murió súbitamente una persona, y no encontramos respuesta, quien descubra la causa habrá explicado el misterio aun si no tuviéramos ninguna idea de en qué casos el veneno en cuestión causa la muerte o en qué casos la enfermedad descubierta mata.

Si queremos entender mejor el contenido y las razones en favor de la Tesis II, habrá que preguntarnos por la naturaleza de la explicación. ¿Qué es, en efecto, la explicación? No tendría caso intentar responder a priori esta pregunta. El hecho es que la humanidad siempre ha pedido y dado explicaciones, siempre se ha intentado explicar por qué ocurren las cosas que ocurren, por qué son como son las cosas que existen. El filósofo apriorista quiere inventar las condiciones de la verdadera explicación y después decretar que la mayoría o todas las explicaciones que el hombre se ha dado son engaños, explicaciones espurias, pseudoexplicaciones. Este filósofo nos dirá que el hecho de que los hombres sientan psicológicamente la fuerza de una explicación no tiene nada que ver con su carácter de explicación auténtica o espuria. La explicación auténtica es la que cumple con ciertas condiciones objetivas, y esto es independiente de la psicología humana. Pero para quienes no vemos cómo pudiera fundarse a priori una teoría de la explicación, el camino es distinto. Nuestra investigación deberá tomar en cuenta nuestras intuiciones acerca de la explicación. ¿Cuáles son las condiciones objetivas que mueven a los hombres a sentir, que han entendido, que tienen una explica- 
ción de algún suceso? La actitud es psicologista; lo que queremos saber, investigar, es un aspecto de nuestra psicología: qué explicaciones nos convencen, por qué nos convencen. Pero no es solamente psicologista; al describir las condiciones objetivas de una explicación convincente podemos preguntarnos qué ventajas sacamos con explicaciones semejantes, y podemos justificar nuestras propias intuiciones. Tenemos, por otra parte, que las explicaciones variarán en sus condiciones objetivas y en su fuerza explicativa, tendremos tipos de explicación que nos parecerán más fuertes, y que de hecho nos permitirán actuar con mayor seguridad, dadas sus características objetivas. Así, por ejemplo, la explicación nomológico-deductiva nos permite predicciones relativaménte más exactas que muchas de las explicaciones del sentido común que no ofrecen una ley estricta. Pero una cosa es reconocer diferencias entre distintos tipos de explicación y otra es canonizar una forma y excomulgar a las demás: en este campo, como en los otros, el filósofo debe ser católico: no reconocer las distintas formas de explicación es inventar una teoría irreal de la racionalidad que naturalmente dejará importantes zonas del conocimiento y de la vida en manos del irracionalista.

Esta actitud universalista no es necesariamente conservadora ni pone a todas las explicaciones en el mismo nivel. Las intuiciones que queremos respetar y explicar no son sólo intuiciones acerca de la aceptabilidad de un tipo de explicación, sino también intuiciones acerca de la superioridad explicativa de unas formas de explicación frente a otras. Tampoco se piense que esta actitud acepta, en general, las explicaciones que la humanidad ha dado. Una cosa es aceptar su capacidad de explicar y otra es aceptar la verdad de su explanans. Debemos ser capaces de entender por qué la mitología griega era explicativa para los griegos, sin aceptar las explicaciones mitológicas. Si no las aceptamos, esto no se debe a razones de una filosofía primera, sino a que no creemos en la existencia de los agentes divinos de los que dependen esas explicaciones.

La Tesis II nos dice que a veces explicaremos por qué sucedió $e$ si decimos que $c$ lo causó. Ante la pregunta "¿por qué sucedió $e$ ?" una respuesta que aceptamos es "porque ocurrió c". Al formular la Tesis II dije que esto ocurre muchas veces, pero no siempre. El análisis de Davidson nos explicaría la razón: no toda mención de $c$ puede satisfacernos en una explicación. Como hemos visto, podemos referirnos a un suceso en formas infinitamente diversas; pero si esto no afecta el valor de verdad del enunciado, sí afecta su aceptabilidad como explicación. Una forma de explicar esta intuición es afirmar que debemos describir la causa en forma tal que podamos entender que pudo producir el efecto cuyo origen queremos conocer. $\mathrm{Si}$, por ejemplo, una señora me cuenta que su abuelo se suicidó, le pregunto por qué, y me explica: "Porque Pascual Orozco venció a las fuerzas federales en Rellano", no entenderé la conexión y querria tener más datos para verla (por ejemplo, que el abuelo 
en cuestión era el comandante de las fuerzas federales en esa ocasión). Menos explicativa aun, sería la descripción: "Porque ocurrio el acontecimiento en el que estuvo pensando mi prima Irma hace media hora." Hay formas de describir los sucesos que, si bien se refieren a ellos, no nos permiten situarlos en una imagen explicativa de las cosas, no nos permiten relacionarlos en la forma pertinente.

Es posible, sin embargo, distinguir entre la mera explicación causal, en la que simplemente se indica una cadena causal, y la explicación que, además, alude a una posibilidad psicológica. En el primer ejemplo del párrafo anterior, "el abuelo de $x$ se suicidó porque Pascual Orozco venció a las fuerzas federales en Rellano" es una explicación. Es cierto que la explicación nos deja insatisfechos. Quisiéramos, además de saber qué causó el suicidio, averiguar cómo pudo esto ser posible psicológicamente. Quisiéramos saber cómo afectó al abuelo de $x$ ese hecho. Aquí, el problema existe porque no sabemos ni siquiera qué relación pudo haber entre el abuelo de $x$ y la batalla. En otros casos, conoceremos la relación pero nos faltarán datos para entender psicológicamente al agente, aunque tendremos datos independientes para conocer la causa de un acto que permanecerá psicológicamente ininteligible. En el segundo ejemplo, la situación es diferente: "porque ocurrió el acontecimiento en el que estuvo pensando mi prima Irma hace media hora" no nos parece explicativo debido a que la frase denotativa con la que nos referimos a ese suceso no nos permite situarlo en nuestro mapa espacio-temporal del mundo, sino indirectamente, en forma semejante a "el autor del Quijote es la persona que pisó este escalón en $t$ ", y en esa medida nos parecerá que la explicación no nos ayuda a entender.

Habrá, entonces, un sentido de entender por qué ocurrió un suceso que consistirá simplemente en colocarlo dentro de un mapa causal del mundo. Tomemos un ejemplo extremo. Supongamos que tenemos una máquina de Galton. Supongamos que nos interesa saber por qué cierta canica se encuentra en determinado receptáculo de la parte de abajo. La explicación puramente causal nos ofrecerá una historia de esa canica, su trayectoria desde que alguien la soltó en la parte superior de la máquina hasta su caída en el receptáculo correspondiente. Quisiera insistir en que esta explicación no necesita de ninguna ley estricta, ni forma parte de ella el hecho de que la probabilidad de caer, digamos, en el primer receptáculo sea de 1/1,024. Podemos no conocer las probabilidades en esta máquina, ni las leyes físicas deterministas relevantes, y saber cuál fue la cadena causal. Propongo este ejemplo como una parábola de lo que podría ser una explicación puramente causal en la historia. Una crónica causal pura sería simplemente la historia de una trayectoria causal. El historiador, sin embargo, haría mal en limitarse a una crónica de este tipo. Conocemos a veces leyes que conectan unos sucesos con otros; en otras ocasiones, cuando los sucesos son actos humanos (porque 
las cadenas causales en la historia incluyen multitud de sucesos naturales o sociales, y estos últimos no son actos humanos ni están constituidos puramente con actos humanos), disponemos de explicaciones psicológicas. Mal haría, entonces, el historiador en no enriquecer con esos conocimientos su explicación.

Los sucesos que deberán mencionarse en una explicación histórica son probablemente de muy diversa indole. Un acto humano puede causar (dadas ciertas circunstancias económicas y sociales) un suceso económico, que a su vez causará efectos en el medio ambiente, que tendrán otras consecuencias que afectarán la economía, etc.

Este trabajo es sólo un planteamiento muy general; si sus ideas sobre la explicación fuesen vagamente correctas, quedaría mucho por desarrollar. Habría que ver cómo se relacionan unos sucesos con otros: v. gr. la Revolución Francesa con los actos individuales de los franceses en esa época; habría que discutir casos de sobredeterminación y muchas otras cosas. Este trabajo es sólo un planteamiento para comenzar a discutir.

INSTTTUTO DE INVESTIGaciones Filosóficas

Hugo Margán

UnIVERSidad Nacional de MÉxico 